\title{
Quantitative trait loci affecting growth-related traits in wild barley (Hordeum spontaneum) grown under different levels of nutrient supply
}

\author{
IAM Elberse ${ }^{1,4}$, TK Vanhala ${ }^{2}$, JHB Turin ${ }^{1}$, P Stam $^{2}$, JMM van Damme ${ }^{1}$ and PH van Tienderen ${ }^{3}$ \\ ${ }^{1}$ Institute of Ecology (NIOO), Boterhoekse straat 48, PO Box 40, 6666 ZG Heteren, The Netherlands; ${ }^{2}$ Department of Plant Breeding, \\ Wageningen University, Bode 28, Postbus 386, 6700 AJ Wageningen, The Netherlands; ${ }^{3}$ Institute for Biodiversity and Ecosystem \\ Dynamics (IBED), University of Amsterdam, PO Box 94062, 1090 GB Amsterdam, The Netherlands
}

The genetic basis of phenotypic plasticity of relative growth rate (RGR), its components and associated morphological traits was studied in relation to nutrient limitation. In all, $140 \mathrm{~F}_{3}$ lines from a cross, made between two Hordeum spontaneum (wild barley) accessions sampled in Israel, were subjected to growth analysis under two nutrient levels. Quantitative trait loci (QTLs) were detected for RGR and three of its components, leaf area ratio (LAR), specific leaf area and leaf mass fraction (LMF). Indications for close linkage (potential pleiotropy) were found, for example, for LAR and LMF. An interesting case is on chromosome 6, at which QTLs for RGR and seed mass were detected in the same region. These QTLs had opposite additive effects, supporting earlier results that plants growing from lighter seeds had a higher RGR. Only two QTLs were significant under both nutrient conditions, suggesting large $Q T L \times$ environment interactions for most traits. For 21 out of 26 QTLs, however, the additive genetic effect was of identical sign in both nutrient environments, but reached the significance threshold in only one of them. Nevertheless, some QTLs detected in one of the two environments had virtually no effect in the other, and QTLs for plasticity were detected for RGR, LAR and LMF, as well as for some morphological traits. QTLs with opposite effects under high and low nutrients were not found. Thus, at the genetic level, there was no evidence for a trade-off between faster growth at high versus low nutrient levels.

Heredity (2004) 93, 22-33, advance online publication, 28 April 2004; doi:10.1038/sj.hdy.6800467

Keywords: QTL; plasticity; nutrient limitation; relative growth rate; wild barley; QTL $\times$ environment interaction

\section{Introduction}

Plant species display large inherent differences in relative growth rate (RGR, net increase in biomass per unit biomass per unit of time), when grown under optimal conditions (Grime and Hunt, 1975; Poorter and Remkes, 1990; Shipley and Peters, 1990; Hunt and Cornelissen, 1997). Species originating from favourable environments tend to have an inherently high maximum RGR, whereas those from less favourable habitats often show an inherently low maximum RGR (Grime and Hunt, 1975; Poorter and Remkes, 1990). In looking for a functional explanation for these findings, RGR is usually broken down into component parts. It is the product of net assimilation rate (NAR, dry mass gain per unit leaf area per day) and leaf area ratio (LAR, ratio of leaf area and total plant mass). LAR is the product of specific leaf area (SLA, ratio of leaf area and leaf mass) and leaf mass fraction (LMF, fraction of total plant mass allocated to the leaves) (Evans, 1972). As yet, the genetic basis of RGR and its components is hardly known. Do a few genes with pleiotropic effects control these components? For

Correspondence: JMM van Damme, Institute of Ecology (NIOO), Boterhoekse straat 48, PO Box 40, 6666 ZG, Heteren The Netherlands. E-mail: j.vandamme@nioo.knaw.nl

${ }^{4}$ Current address: Applied Plant Research, Nursery Stock Research Unit, PO Box 118, 2770 AC Boskoop, The Netherlands.

Received 17 December 2002; accepted 2 November 2003; published online 28 April 2004 example, genes that affect early developmental characters like seed mass, or hormone activity and sensitivity. Or has natural selection brought together the genes that independently affect the components associated with an inherently high or low growth rate?

Natural conditions are seldom optimal for plant growth, so it is important to understand how plant growth responds to variation in environmental factors (Chapin III, 1991; Lambers et al, 1998). Nutrient availability is an important factor in limiting plant production, and, obviously, growth reduction by nutrient limitation is hard to avoid. Nevertheless, plants react by changing their growth characteristics and morphology to minimise the effects of nutrient limitation, for instance, by increasing allocation to roots (Reynolds and D'Antonio, 1996; Poorter and Nagel, 2000). Thus, plants may respond to nutrient limitation by phenotypic plasticity, and the nature of this response is subject to natural selection. As yet, knowledge of the genes underlying genotype $\times$ environment interactions ('plasticity genes') is limited. For instance, genes affecting the plasticity of a trait could differ from those influencing the average phenotype (Via et al, 1995).

The present study aims to address two main questions. First, what is the genetic basis of RGR and related traits under suboptimal conditions, and, in particular, how does nutrient level affect the expression of the genes? Second, are genes for plasticity present and where are these located? Since the traits of interest are quantitative 
by nature, these questions were addressed by quantitative trait locus (QTL) analysis (Paterson et al, 1988; Mauricio, 2001).

As a model species, Hordeum spontaneum C. Koch. was used, the wild ancestor of cultivated barley (Hordeum vulgare L.) (Zohary, 1969). Owing to the close relationship between $H$. spontaneum and $H$. vulgare, it was expected that the molecular techniques developed for cultivated barley (one of the model species for molecular genetic studies in monocots, Yin et al, 1999b) could be easily implemented for wild barley. Several molecular marker maps are available for cultivated barley (eg Becker et al, 1995; Qi et al, 1998; Yin et al, 1999b). In addition, various QTL studies have been performed on this species, mainly on agronomic quantitative traits (eg Powell et al, 1997; Yin et al, 1999a, b; Teulat et al, 2001).

The QTL analysis was performed on a cross between $H$. spontaneum plants from two Israeli accessions differing in growth-related traits. The following specific questions were asked:

- Where are genes that regulate RGR and its components located?

- Is variation in RGR related to seed mass?

- Is pleiotropy potentially a factor causing the association among traits and/or are more independent QTLs involved?

- Can QTLs responsible for variation in plasticity be identified, and, if so, do they differ from QTLs affecting the average phenotype?

\section{Materials and methods}

\section{The study species}

$H$. spontaneum is an annual diploid species $(2 n=14)$ (Nilan, 1964) and is predominantly self-fertilising (Brown et al, 1978). This species is widely distributed over the eastern Mediterranean basin and in Western Asiatic countries, where it occurs over a wide range of climates and soils (Zohary, 1969; Nevo, 1992). Israel represents a large portion of the ecological range of wild barley (Nevo et al, 1984). Natural populations from Israel and Iran show genetic variation, both among and within populations, for growth as well as associated morphological traits (Van Rijn et al, 2000; Elberse et al, 2003). Genetic differences in plasticity for the RGR components LMF and LAR have been shown both among populations and among accessions-within-populations (Elberse et al, 2003).

\section{Plant material}

From a set of natural wild barley populations, sampled by Nevo et al (1984), one accession from Ashqelon (Nevo no. 28-77) and one from Mehola (Nevo no. 22-28) were crossed. The Mehola accession has the higher growth rate (Elberse et al, 2003; Van Rijn et al, 2000). Ashqelon is a coastal site, whereas Mehola is situated in the Jordan Valley. The habitats differ in rainfall, humidity, evaporation and soil type (Nevo et al, 1984). The Mehola soil is more fertile than the Ashqelon soil (KJF Verhoeven, unpublished data). From the 'Ashqelon' $\times$ 'Mehola' cross, eight $F_{1}$ plants were self-fertilised. Subsequently, the $F_{2}$ plants were selfed, bulking the seeds per plant, resulting in eight subfamilies of $\mathrm{F}_{2}$-derived $\mathrm{F}_{3}$ lines. The size of the subfamilies ranged from 14 to 43 lines, with a total of $233 \mathrm{~F}_{3}$ lines.

Genetic linkage map

A total of $233 \mathrm{~F}_{2}$ plants from these subfamilies were scored for 13 SSR markers and 22 ALFP primer combinations, and a molecular map was constructed by Poorter et al (submitted). Due to unexpected heterozygosity of one of the parents, the size of this marker map was estimated at $445 \mathrm{cM}$, which only covers $55 \%$ of the entire genome. Chromosome numbers were assigned to the linkage groups using SSR loci (Ramsay et al, 2000) and AFLP markers that were in common with earlier maps. These earlier maps were based on the following crosses of cultivated barley: 'L94' × 'Vada' (Qi et al, 1998), 'L94' $\times$ '115-6' (P Lindhout, personal communication), 'Apex' $\times$ 'Prisma' (Yin et al, 1999b) and 'Proctor' $\times$ 'Nudinka' (Becker et al, 1995).

As clustering of markers was clearly manifest on the linkage map, for the purpose of QTL mapping, a 'core' map has been made with relatively uniform coverage. Where possible the markers in the core map were chosen at about $4 \mathrm{cM}$ intervals, retaining the most informative markers (ie scored co-dominantly and with the least number of missing values). This core map contained 96 markers, covering $437 \mathrm{cM}$.

\section{Growth analysis}

A randomised complete block design was carried out, including seven blocks, $140 \mathrm{~F}_{3}$ lines from the 'Ashqelon' $\times$ 'Mehola' cross (randomly chosen from the lines for which enough seeds were available), two nutrient levels and two harvest times. Each block contained one replicate. For practical reasons, blocks could not be handled simultaneously. Thus, these were carried out one by one, being replicates at different moments in time. Blocks had to be divided over two climate chambers. To this end, two groups of lines were composed, which alternately grew in one or the other climate chamber.

In order to break dormancy, caryopses were given a period of 9 weeks of after-ripening at $38^{\circ} \mathrm{C}$ in a drying oven. To stimulate germination, the glumellae, lemmas and spikelet stalks were removed from the caryopses (Gutterman et al, 1996). Eight naked caryopses per accession per block were weighed together and the average mass per seed was calculated. Subsequently, they were germinated at $4{ }^{\circ} \mathrm{C}$ in the dark, in Petri dishes with filter paper moistened by demineralised water. After 7 days, the Petri dishes were transferred to an incubator (day/night temperature: $22^{\circ} \mathrm{C} / 16^{\circ} \mathrm{C}$, day length: $16 \mathrm{~h}$, light intensity: varied between 21 and $\left.120 \mu \mathrm{mol} \mathrm{m}^{-2} \mathrm{~s}^{-1}\right)$. After 2 days, the young plants were transferred to a climate chamber (day/night temperature: $23^{\circ} \mathrm{C} / 15^{\circ} \mathrm{C}$, day length: $16 \mathrm{~h}$, light intensity at plant level varying between 289 and $406 \mu \mathrm{mol} \mathrm{m} \mathrm{m}^{-2} \mathrm{~s}^{-1}$, relative humidity: $80 \%$ ) for the growth experiment. Plants grew individually in pots containing coarse quartz sand without any organic matter.

A modified Hoagland's solution (see Elberse et al, 2003) was administered as source for water and nutrients. By dilution with demineralised water, 1/8 and 1/512 strength Hoagland's solutions were produced. Immediately after planting, approximately $100 \mathrm{ml}$ of this solution was carefully given by hand. From the next day 
onwards, Hoagland's solution was supplied by a computerised irrigation system. The first day after planting, each plant received $80 \mathrm{ml}$ twice and during the remainder of the experiment $80 \mathrm{ml}$ was supplied once a day (see Elberse et al, 2003). At 2 days after planting, the relative humidity was lowered to $60 \%$ and kept at this level until the end of the experiment.

At 18 and 28 days after sowing, plants were harvested in order to determine RGR and NAR. At each harvest, the number of tillers, leaves and roots were counted and the length and width of the longest leaf were measured for all plants. A digital image analysis system (Delta-T Devices Ltd, Cambridge, UK) was used to measure the total leaf area per plant. Plants were separated into leaf blades, leaf sheaths and roots, oven-dried for at least $48 \mathrm{~h}$ at $70^{\circ} \mathrm{C}$, after which dry mass was measured.

The LAR, SLA and LMF were calculated per plant. To calculate RGR and NAR, the plants of the first and the second harvest were paired; each pair belonged to the same block, nutrient treatment and $F_{3}$ line. Due to reallocation, some plants had dead leaf parts, especially in the low nutrient treatment. Green and dead leaf parts were weighed separately and the leaf area of only green leaf parts was measured. In the formulae, the total plant dry mass included dead leaf parts, as these are part of the biomass produced by the plant. At the time of harvest, however, these dead leaf parts were not part of the photosynthetic system and, therefore, were not included in leaf mass or leaf area used in the equations.

\section{Data analysis}

To correct for climate chamber $\times$ block effects, for each trait the mean value per climate chamber per block was subtracted from each plant value. Then the means per line per nutrient level were calculated. These corrected data were used in all subsequent analyses. For seed mass no such correction was applied, as it was measured before the start of the experiment.

ANOVA was performed, using the GLM procedure (Type III EMS) in SAS for Windows, release 8.02. The class variables were nutrient level, time of harvest and $F_{3}$ line (abbreviated to $\mathrm{N}, \mathrm{T}$ and $\mathrm{L}$, respectively). The line and all interactions with it were treated as random factors.

A measure for plasticity was calculated, using the environmental sensitivity method (Falconer and Mackay, 1996). In this method, the mean values per $F_{3}$ line per nutrient level were regressed on the overall mean values per nutrient level. The slope of a regression line represents the environmental sensitivity or plasticity of an $\mathrm{F}_{3}$ line.

QTL analyses (MapQTL version 4.0, http:// www.plant.wageningen-ur.nl/products/mapping/right. $\mathrm{htm}$ ) were performed for the mean seed mass, for all other traits for each nutrient level separately (using the means per line per nutrient level) and for plasticity values. Initially, interval mapping was performed. Subsequently, multiple-QTL model (MQM) analysis (Jansen and Stam, 1994, also known as composite interval mapping, Lynch and Walsh, 1999) was performed, using markers near LOD peaks $>2.0$ as covariates. Thus, the power and precision of QTL mapping was increased. If new peaks appeared, these were also employed as a cofactor until no further cofactor effect was found.
Cofactors without an effect were left out again. The set of cofactors that was in use when the LOD profile stabilised was subsequently used in restricted MQM mapping. This method uses all selected cofactor markers, except the ones on the linkage group that a QTL is fitted on. In QTL analysis, the mapping step size was $5 \mathrm{cM}$ and dominance was fitted. An LOD significance threshold of 2.7 was applied for defining a QTL. This threshold value was calculated according to the method of Van Ooijen (1999).

\section{Results}

A higher nutrient level resulted in higher line means for all traits (Figure 1). Histograms for high and low nutrients were completely separate from each other for all traits except NAR and SLA, where some overlap occurred. Transgression of the $\mathrm{F}_{3}$ lines was shown for most traits. Number of tillers at the high nutrient level, leaf width at both nutrient levels and seed mass were the exceptions. The seed mass of the 'Ashqelon' parent was strikingly higher than that of all $\mathrm{F}_{3}$ lines. Possibly, an unintended selection for low seed mass took place, as $F_{2}$ plants used for QTL analysis had to have enough seeds for measuring the phenotypic traits in the $\mathrm{F}_{3}$. Of the $237 \mathrm{~F} 2$ lines available, only 150 had enough seeds and the 140 lines used were randomly taken from those 150 . We observed that plants had either many small seeds or fewer large seeds (data not shown). Thus, a trade-off may exist between the seed number produced per plant and the individual mass of the produced seeds, which may be responsible for this result.

Highly significant nutrient and harvest time effects were found for all characteristics (Table $1 \mathrm{a}$ and $\mathrm{b}$ ). Line effects, however, were only present for the number of roots, leaf length and leaf width. Differences in plasticity among lines in reaction to nutrient limitation, represented by significant nutrient $\times$ line effects, were detected only for LAR, LMF and leaf width. The seed mass ANOVA (not shown) indicated that the $F_{3}$ lines differed significantly in seed mass $\left(\mathrm{F}_{[139,840]}=11.96^{* * *}\right)$.

Significant $\mathrm{N} \times \mathrm{T} \times \mathrm{L}$ interactions were also observed (Table 1), indicating that the plasticity of lines in response to nutrient levels differed for the two harvests. Initial differences among lines were small (eg the number of tillers at the first harvest), but tended to increase with plant age and nutrient supply.

\section{QTLS}

In all, 11 QTLs were found for RGR, LAR, SLA and LMF (Table 2a, Figure 2) spread over the genome. LOD scores varied from the significance threshold 2.7 up to 6.0 , and percentage of variance explained by a QTL varied from 6.8 to 18.1 . The variance explained by all QTLs per trait per nutrient level together ranged from 9.7 to $27.3 \%$ of the total variance among lines. No QTLs were detected for NAR. The lines were coded such that a positive effect represents a difference in the direction of the 'Ashqelon' parent, and a negative additive effect a difference in the direction of the 'Mehola' parent. Negative additive effects were found for all of these QTLs, except for the QTL for SLA (both nutrient levels) on chromosome $3 \mathrm{a}$. This means that for this SLA-QTL the 'Ashqelon' allele had a positive effect on the SLA mean, 
a

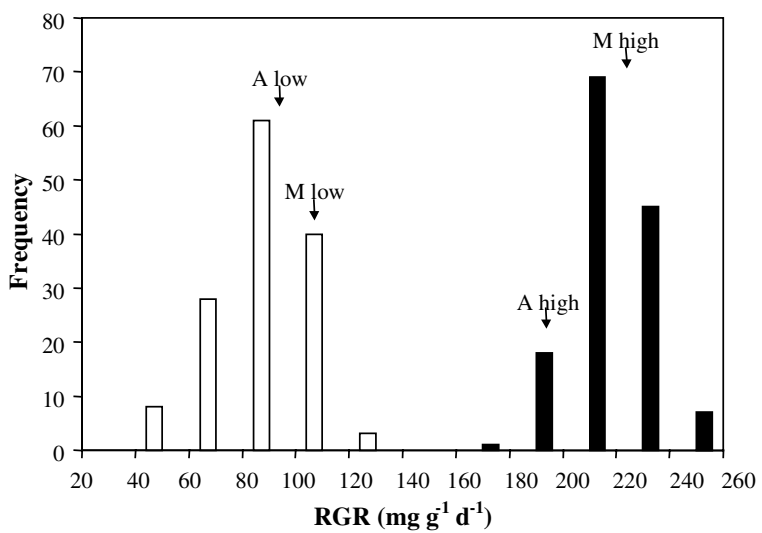

Low nutrient

High nutrient

Seed mass
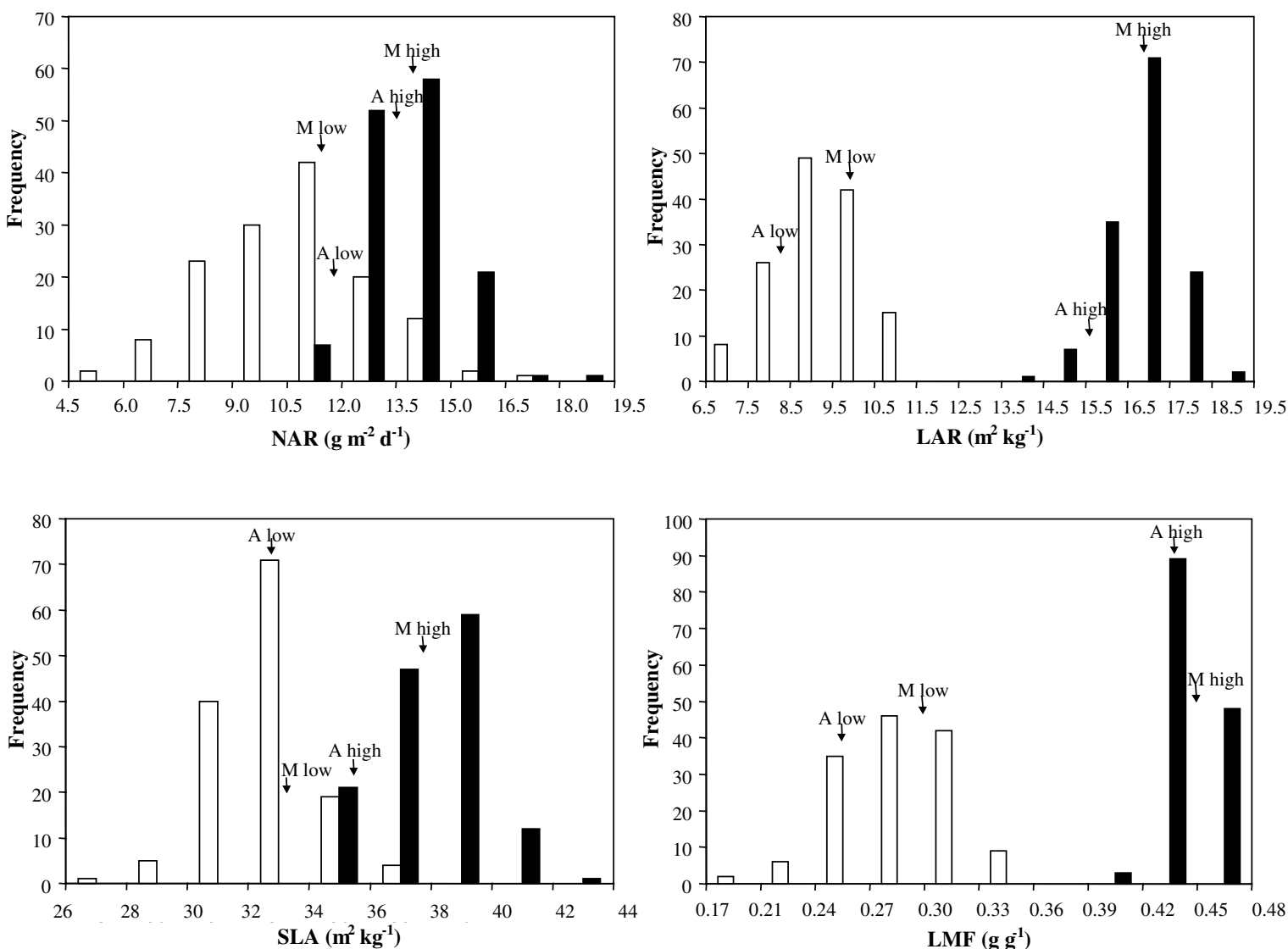

Figure 1 (a) Histograms on line means per nutrient level for RGR and its components. The average value for the parents 'Ashqelon' (A) and 'Mehola' (M) at both low and high nutrient levels is indicated with arrows. (b) Histograms on line means for seed mass (Seedm) and on line means per nutrient level for the number of tillers (\# tillers), number of leaves (\# leaves), number of roots (\# roots), leaf length (Leafl) and leaf width (Leafw). The average value for the parents 'Ashqelon' (A) and 'Mehola' (M) at both low and high nutrient levels is indicated with arrows. For seed mass, no different nutrient levels were applied.

but for RGR, LAR and LMF the 'Mehola' allele increased the trait mean, hence contributing to the observed difference in growth characters between the two parental lines.

QTLs were detected for all morphological traits (Table 2B, Figure 2). LOD scores ranged from 2.8 to 5.5 and the percentage of variance explained by a QTL varied from 6.1 to 23.1. Summarised over all QTLs per trait per nutrient level, the variance explained ranged from 8.5 to $35.4 \%$ of the total variance. The highest numbers of QTLs were found for the number of leaves (five) and leaf length (six), while only one QTL was detected for number of tillers. Both positive and negative additive effects were observed.

MapQTL output provides no standard errors for the additive and dominance effects, and, therefore, care should be taken when interpreting these values. Dominance effects for RGR and components as well as for 
b
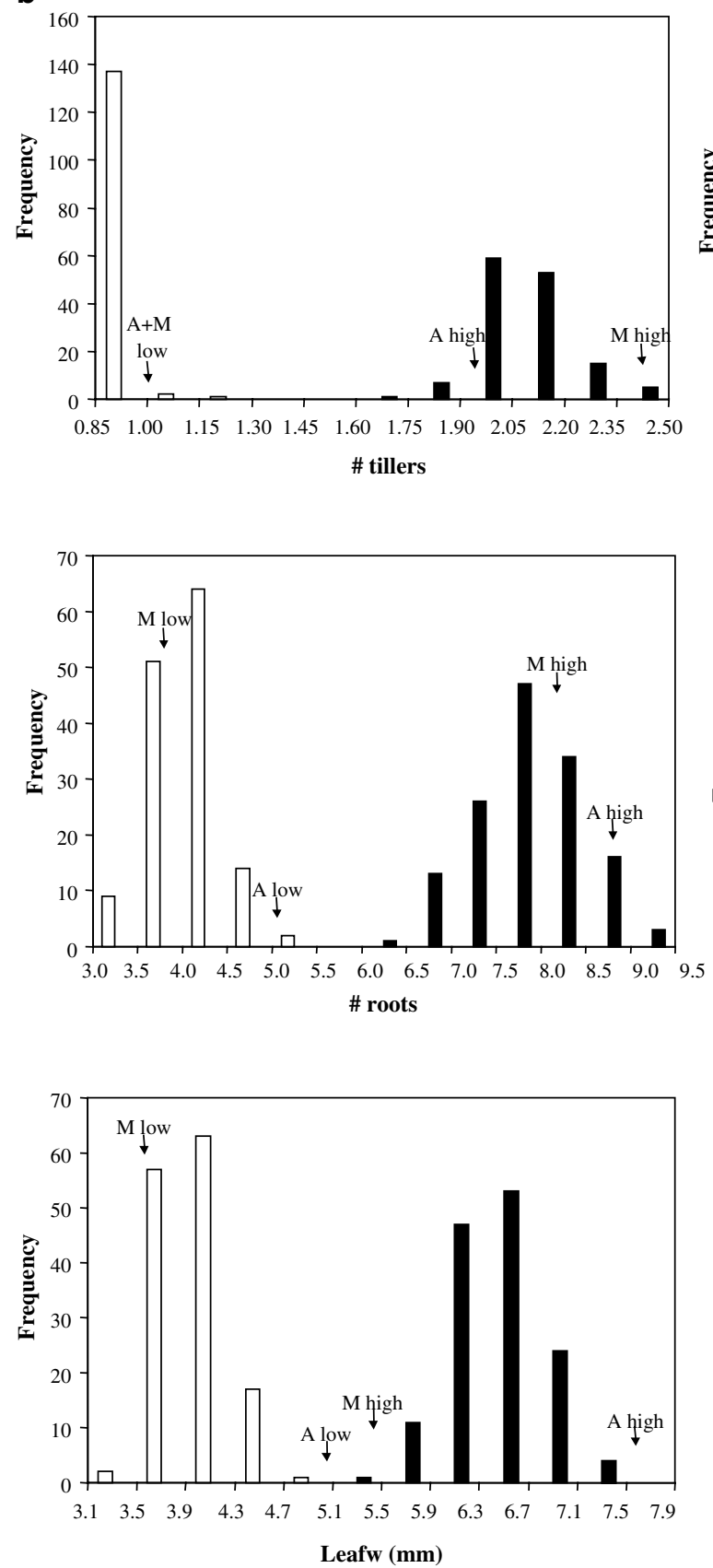

Figure 1 (Continued)

morphological traits ranged from close to zero to a value larger than the additive effect. This suggests that the mode of gene action ranged from complete additivity (eg LAR on chromosome 5) to overdominance (eg LMF on chromosome 4).

\section{Association among traits}

Support intervals of QTLs for LAR and LMF were frequently overlapping (Figure 2, chromosomes 2a, 4 and 5). On chromosome 4, a QTL for SLA at the low nutrient level coincides with QTLs for LAR and LMF, both at high nutrient conditions. Both QTLs for SLA on chromosome
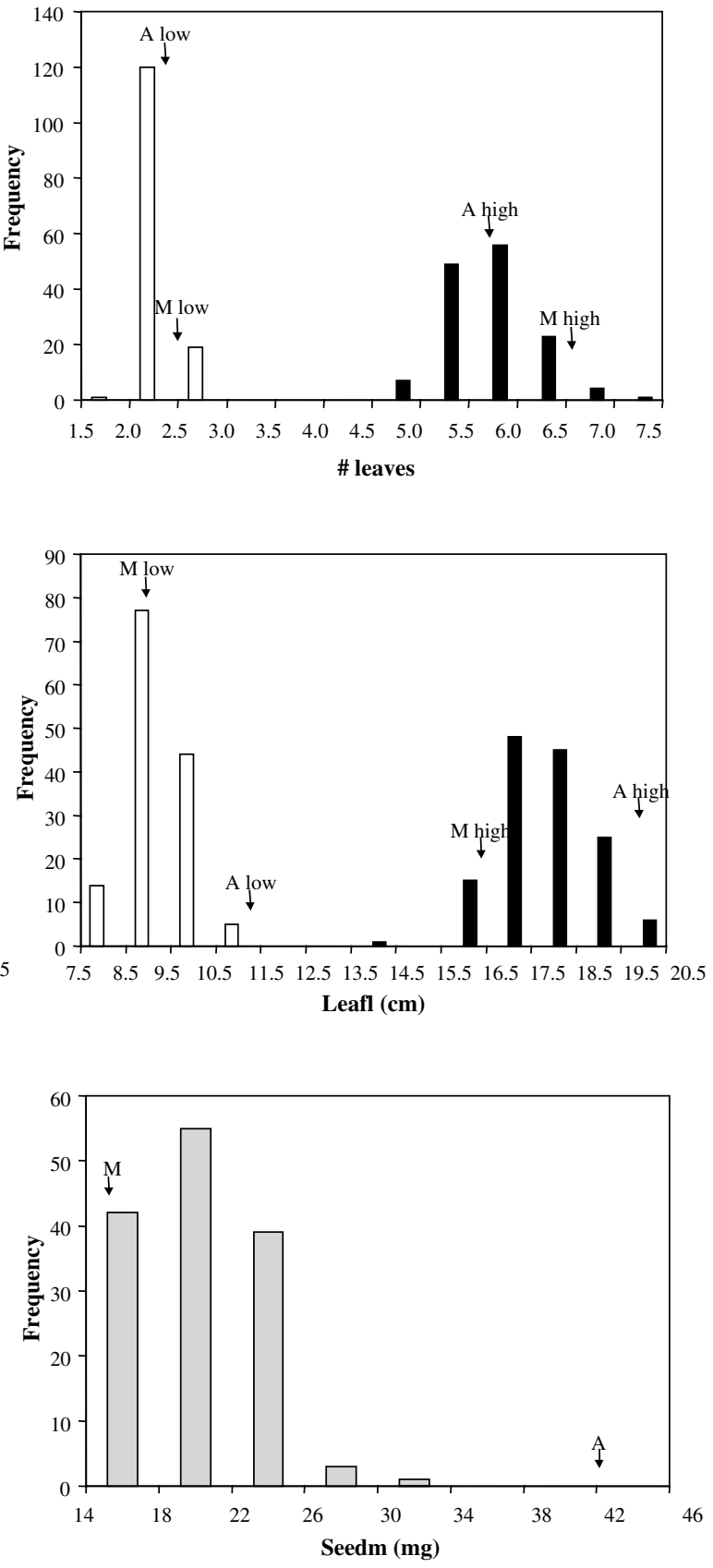

3a and RGR on chromosome 6 show no overlap with any other RGR components. Many morphological traits also showed overlapping QTL support intervals. For example, on chromosomes 1 and 2a, QTLs for the number of leaves and leaf length show overlap and on chromosomes 6 and 7 coinciding QTLs are found for the number of leaves and number of roots.

In addition, overlapping QTL intervals were found for RGR and components on the one hand and morphological traits on the other on chromosomes $2 \mathrm{a}, 4,5$ and 6 . The RGR-QTL on chromosome 6, for example, overlaps with QTLs for the number of leaves, number of roots, leaf width and seed mass. 
Table 1 ANOVA results (data corrected for climate chamber $\times$ block effect) for (a) RGR and its components, and (b) for the morphological traits

\begin{tabular}{|c|c|c|c|c|c|c|}
\hline Effect & d.f. & $R G R$ & NAR & $L A R$ & $S L A$ & $L M F$ \\
\hline \multicolumn{7}{|l|}{ (a) } \\
\hline $\mathrm{N}$ & 1 & $5265^{* * *}$ & $220^{* * *}$ & $5696^{* * *}$ & $911^{* * *}$ & $4247^{* * *}$ \\
\hline $\mathrm{T}$ & 1 & - & - & $963^{* * *}$ & $3795^{* * *}$ & $45.6^{* * *}$ \\
\hline $\mathrm{L}$ & 139 & 1.26 & 0.96 & 0.66 & 1.24 & 1.12 \\
\hline $\mathrm{N} \times \mathrm{T}$ & 1 & - & - & $234^{* * *}$ & 0.13 & $61.9^{* * *}$ \\
\hline $\mathrm{N} \times \mathrm{L}$ & 139 & 1.02 & 1.10 & $1.33^{*}$ & 1.13 & $1.51^{* *}$ \\
\hline $\mathrm{T} \times \mathrm{L}$ & 139 & - & - & $1.70^{* *}$ & 1.19 & $1.36^{*}$ \\
\hline $\mathrm{N} \times \mathrm{T} \times \mathrm{L}$ & 139 & - & - & 1.60 & 1.15 & $1.74^{* * *}$ \\
\hline \multicolumn{7}{|l|}{ (b) } \\
\hline Effect & d.f. & \#tillers & \#leaves & \#roots & Leafl & Leafw \\
\hline $\mathrm{N}$ & 1 & $9252^{* * *}$ & $8185^{* * *}$ & $7242^{* * *}$ & $12196^{* * *}$ & $7642^{* * *}$ \\
\hline $\mathrm{T}$ & 1 & $9383^{* * *}$ & $10316^{* * *}$ & $6843^{* * *}$ & $11118^{* * *}$ & $1129^{* * *}$ \\
\hline $\mathrm{L}$ & 139 & 1.00 & 1.24 & $1.95^{* *}$ & $2.44^{* * *}$ & $2.10^{* * *}$ \\
\hline $\mathrm{N} \times \mathrm{T}$ & 1 & $9251^{* * *}$ & $5828^{* * *}$ & $5164^{* * *}$ & $5585^{* * *}$ & $3783^{* *}$ \\
\hline $\mathrm{N} \times \mathrm{L}$ & 139 & 1.00 & 1.19 & 1.14 & 1.12 & $1.44^{*}$ \\
\hline $\mathrm{T} \times \mathrm{L}$ & 139 & 1.00 & 1.09 & 1.18 & 0.82 & $1.39^{*}$ \\
\hline $\mathrm{N} \times \mathrm{T} \times \mathrm{L}$ & 139 & $1.54^{* * *}$ & $1.80^{* * *}$ & 1.18 & $1.75^{* * *}$ & $1.44^{* * *}$ \\
\hline
\end{tabular}

Trait abbreviations are as in Figure 1. F values are presented and the asterisks indicate significance. $\mathrm{N}=$ nutrient level, $\mathrm{T}=$ time of harvest and $\mathrm{L}=\mathrm{F}_{3}$ line. The residual d.f.'s are 1659 for RGR, 1655 for NAR and 3336 for LAR, SLA and LMF.

Table 2 QTLs detected for (a) RGR and components and (b) for morphological traits

\begin{tabular}{|c|c|c|c|c|c|c|c|}
\hline Trait & Nutrient level & Chromosome & Position (cM) & LOD score & $\%$ variance explained & Additive effect & Dominance effect \\
\hline \multicolumn{8}{|l|}{ (a) } \\
\hline RGR & Low & 6 & 11.7 & 3.4 & 9.7 & -0.46 & 0.61 \\
\hline RGR & High & 6 & 11.7 & 4.2 & 13.9 & -0.53 & -0.02 \\
\hline \multirow[t]{2}{*}{ LAR } & Low & $2 a$ & 35.4 & 2.9 & 9.6 & -0.41 & -0.34 \\
\hline & & 5 & 45.0 & 5.8 & 17.7 & -0.63 & 0.05 \\
\hline LAR & High & 4 & 5.3 & 2.7 & 10.3 & -0.53 & 0.17 \\
\hline \multirow[t]{2}{*}{ SLA } & Low & $3 a$ & 52.6 & 6.0 & 18.1 & 0.63 & 0.01 \\
\hline & & 4 & 23.7 & 3.0 & 7.2 & -0.43 & -0.13 \\
\hline SLA & High & $3 a$ & 52.6 & 4.0 & 12.5 & 0.40 & -0.83 \\
\hline \multirow[t]{2}{*}{ LMF } & Low & $2 a$ & 29.6 & 2.8 & 6.8 & -0.34 & -0.35 \\
\hline & & 5 & 37.3 & 5.0 & 16.4 & -0.50 & -1.13 \\
\hline LMF & High & 4 & 26.4 & 3.4 & 9.8 & -0.37 & 1.04 \\
\hline \multicolumn{8}{|l|}{ (b) } \\
\hline \#tillers & High & 4 & 42.8 & 3.4 & 10.2 & -0.34 & -0.89 \\
\hline \#leaves & Low & 1 & 3.0 & 2.8 & 8.5 & 0.40 & -0.41 \\
\hline \multirow[t]{4}{*}{ \#leaves } & High & $2 \mathrm{a}$ & 29.6 & 3.4 & 8.5 & -0.32 & -0.61 \\
\hline & & 4 & 42.8 & 3.4 & 7.9 & -0.40 & -0.48 \\
\hline & & 6 & 15.7 & 2.9 & 6.1 & -0.36 & -0.36 \\
\hline & & 7 & 14.3 & 3.7 & 9.5 & -0.39 & -0.31 \\
\hline \multirow[t]{2}{*}{ \#roots } & High & 6 & 11.7 & 3.2 & 7.5 & -0.38 & -0.44 \\
\hline & & 7 & 14.3 & 4.7 & 11.6 & -0.48 & 0.05 \\
\hline \multirow[t]{3}{*}{ Leafl } & Low & $2 a$ & 1.7 & 5.5 & 13.1 & 0.58 & 0.27 \\
\hline & & 4 & 5.0 & 5.4 & 14.6 & 0.61 & 0.48 \\
\hline & & 5 & 49.8 & 3.2 & 7.7 & -0.29 & -0.91 \\
\hline \multirow[t]{3}{*}{ Leafl } & High & 1 & 31.4 & 2.8 & 7.4 & -0.35 & -0.48 \\
\hline & & $2 b$ & 9.3 & 3.3 & 8.6 & 0.30 & -0.91 \\
\hline & & 5 & 0.0 & 2.9 & 9.9 & 0.43 & -0.54 \\
\hline \multirow[t]{2}{*}{ Leafw } & Low & $2 a$ & 1.7 & 3.2 & 10.3 & 0.39 & -0.64 \\
\hline & & 6 & 5.0 & 5.3 & 23.1 & 0.74 & -0.69 \\
\hline Leafw & High & 4 & 14.0 & 3.5 & 12.1 & 0.36 & 0.89 \\
\hline \multirow[t]{2}{*}{ Seedm } & - & 5 & 56.4 & 3.0 & 9.1 & 0.17 & -1.12 \\
\hline & & 6 & 8.1 & 2.9 & 8.4 & 0.47 & -0.18 \\
\hline
\end{tabular}

Traits abbreviated are as in Figure 1. Only significant QTLs are shown. The QTL position is that of peak LOD score. \% variance explained $=$ percentage phenotypic variance explained by the QTL. Additive effect $=\left(\bar{x}_{\mathrm{AA}}-\bar{x}_{\mathrm{BB}}\right) / 2$; dominance effect $=\left(\bar{x}_{\mathrm{AB}}-\left(\bar{x}_{\mathrm{AA}}+\bar{x}_{\mathrm{BB}}\right) /\right.$ $2) \times 2$. Both the additive and dominance effects are given as (Ashqelon-Mehola) values and were standardised by dividing by the standard deviation of all line means. 
Chromosome 1

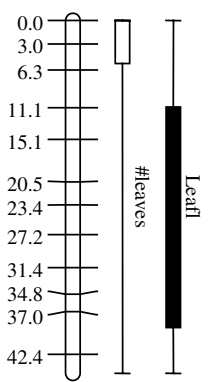

Chromosome 2a

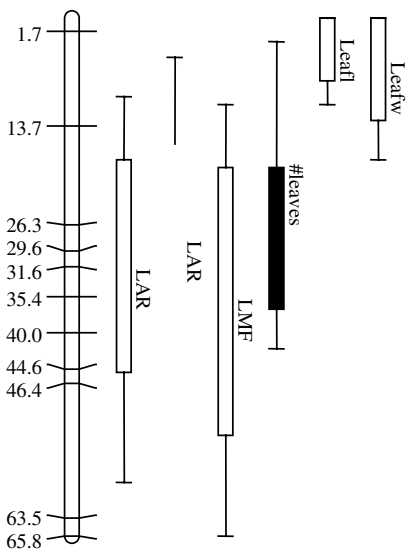

Chromosome 2b

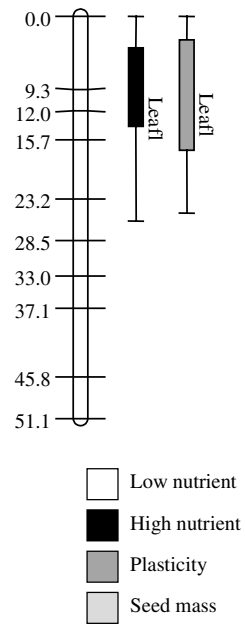

Chromosome 3a

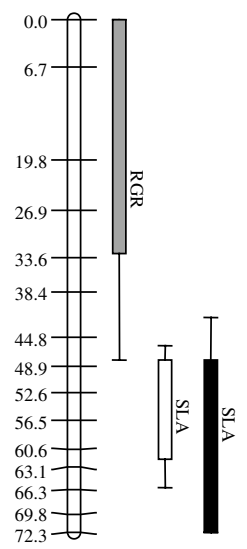

Chromosome 5
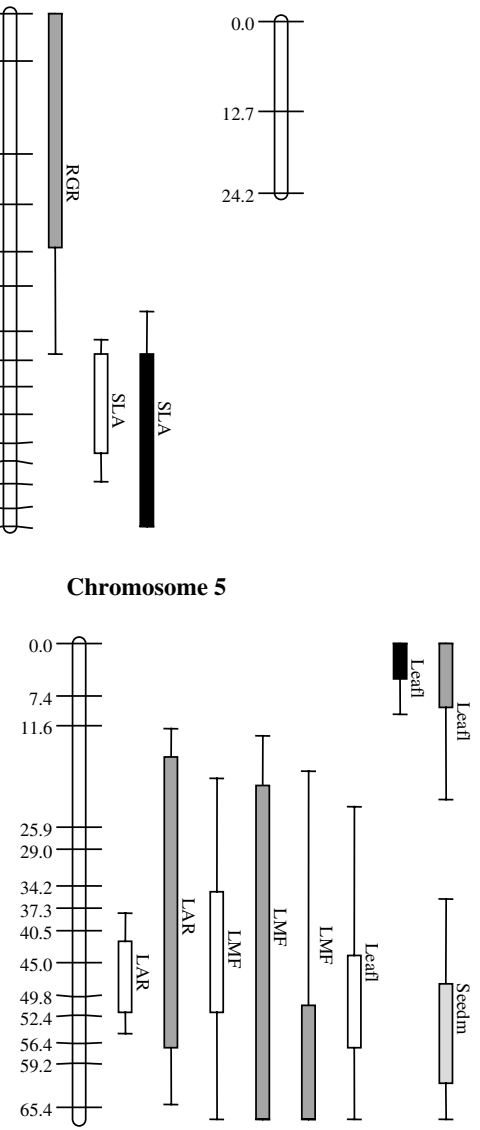

Chromosome 4
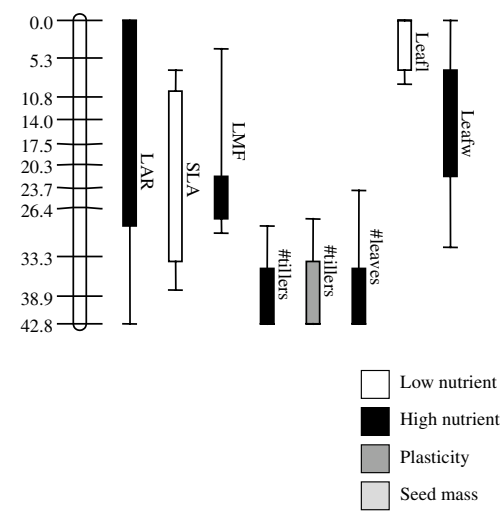

Chromosome 6

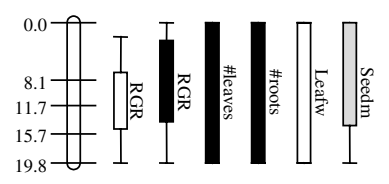

Chromosome 7

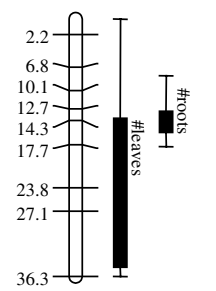

Low nutrient

High nutrient Plasticity

Seed mass

Figure 2 Linkage map (core map) including significant QTLs for low and high nutrient levels, plasticity and seed mass. Bars indicate the 90\% support interval and whiskers indicate the $95 \%$ support interval, obtained by LOD 1 and LOD 2 drop, respectively. For trait abbreviations, see the legend to Figure 1.

Effect of nutrient level on QTLs

For only two traits were significant QTLs found in the same region for both nutrient levels: SLA on chromo- some 3a and RGR on chromosome 6 (Figure 2). As the QTL support intervals for leaf length at both nutrient levels on chromosome 5 show no overlap, these are 
considered to be two separate QTLs. Thus, in general, different QTLs were found in the two different environments. From Figure 2, however, it is not clear whether the QTLs not found are really not there or are peaks below the significance level. To gain more insight into these patterns, the additive effects in the two nutrient environments were plotted against each other for those loci at which a significant QTL (peak LOD) was found in at least one environment (Figure 3). The four quadrants in this graph represent different types of genes, either affecting a trait in the same direction at both nutrient levels (quadrants I and III), or in opposite directions (quadrants II and IV). Furthermore, QTLs that affect a trait in one environment only are expected to appear close to the figure's axes. This 'quadrant analysis' shows that the additive effects of corresponding map positions in both environments mostly have the same sign (Figure 3). Out of 26 QTLs, 21 had additive effects with identical signs in the two environments and only five had opposite signs (deviation from a 13:13 ratio: $\chi^{2}=9.8$, d.f. $=1, P<0.01)$. Since 'Mehola' is known to grow faster than 'Ashqelon', negative additive effects are expected to be more common for growth-related traits. Indeed, 'Mehola' alleles at QTLs had a positive effect in both environments on RGR and LMF, as well as on the number of tillers, number of leaves and number of roots (quadrant III). QTLs for which the 'Ashqelon' alleles had a positive effect in both environments are SLA, number of leaves, leaf length and leaf width (quadrant I). No clear opposite additive effects across environments were found. Some data points appeared close to one of the axes, such as the three LAR data points. Thus, 'Mehola' alleles positively influenced this trait in one environment, whereas they had little or no effect in the other.

\section{QTLs for variation in phenotypic plasticity}

QTLs for plasticity, measured as the slope of environmental sensitivity graphs, were found for RGR, LAR, LMF, number of tillers and leaf length (Table 3, Figure 2). The gene action of the plasticity QTLs also varied from complete additivity to overdominance. Figure 2 shows that a QTL for plasticity is usually found at the same region as a QTL for the trait at one of the nutrient levels. The only exception to this trend is the QTL for plasticity of RGR on chromosome 3a (Table 3).

The two overlapping QTLs for LMF on chromosome 5 (Figure 2) are considered to be separate QTLs. The reason is that in MQM analysis a cofactor on either one of these

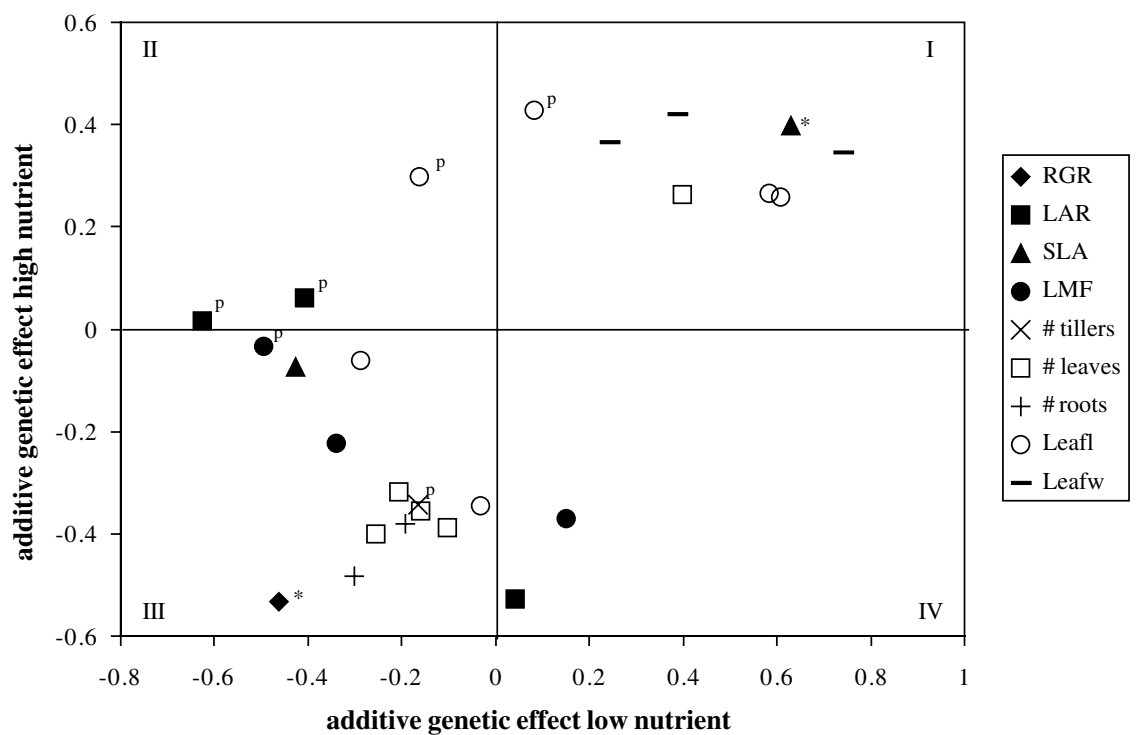

Figure 3 Additive genetic effects of the QTLs, at least significant in one environment, for the low and high nutrient conditions. Positive additive effects indicate that the 'Ashqelon' allele increased the trait mean and negative additive effects represent a positive effect of 'Mehola' on the trait mean. The QTLs that were significant under both conditions are indicated with ${ }^{\prime \prime \prime}$. A ' $p$ ' points to a QTL that was accompanied by a plasticity QTL for the same trait. I-IV mark the quadrants of the graph.

Table 3 QTLs detected for plasticity of RGR and components as well as morphological traits (abbreviated as in Figure 1)

\begin{tabular}{|c|c|c|c|c|c|c|}
\hline Trait & Chromosome & Position (cM) & LOD score & $\%$ variance explained & Additive effect & Dominance effect \\
\hline RGR & $3 a$ & 26.9 & 2.7 & 11.2 & 0.13 & 1.26 \\
\hline \multirow[t]{2}{*}{ LAR } & $2 a$ & 23.7 & 3.1 & 13.3 & 0.39 & 0.91 \\
\hline & 5 & 25.9 & 4.3 & 13.7 & 0.42 & 0.86 \\
\hline \multirow{2}{*}{ LMF } & 5 & 37.3 & 3.8 & 14.3 & 0.40 & 1.21 \\
\hline & 5 & 56.4 & 4.6 & 14.6 & 0.53 & 0.07 \\
\hline \#tillers & 4 & 42.8 & 3.0 & 8.9 & -0.30 & -0.88 \\
\hline \multirow[t]{2}{*}{ Leafl } & 5 & 0.0 & 3.2 & 10.5 & 0.48 & -0.27 \\
\hline & $2 b$ & 9.3 & 3.0 & 7.1 & 0.38 & -0.30 \\
\hline
\end{tabular}

Further legends are as in Table 2. 
peaks did not absorb the variance at the other peak and thus the peaks cannot represent the same QTL.

\section{Discussion}

\section{QTLS}

In contrast to the other growth-related traits, no significant QTLs were found for NAR, and thus no genes could be detected that affected the efficiency of biomass increase per unit leaf area. This could indeed be a biological phenomenon if such a measure of efficiency is always under directional selection, and therefore is expected to exhibit less genetic variation. However, it might also be caused by a relatively high measurement error. For NAR, more measurements (leaf area and plant dry mass, both at two harvest dates), each with its own error, are necessary than for RGR (plant dry made at two harvest times). For LAR and SLA, the same measurements were done as for NAR, but for QTL analysis means over both harvests were used, giving a more precise estimate. NAR, indeed, had the highest coefficient of variation $(38 \%)$ for the error term in comparison to different accessions (see Elberse et al, 2003), and in the present study the standard deviation of the $140 \mathrm{~F}_{3}$ lines for NAR was large at the low nutrient treatment $\left(\right.$ s.d. $=5.6$ and mean $\left.=10.7 \mathrm{~g} \mathrm{~m}^{-2} \mathrm{~d}^{-1}\right)$. This high error variance makes it more difficult to detect a QTL and the failure to do so may have been a matter of power of the QTL analysis rather than a biological pattern.

The QTLs found can be compared to QTLs for the same traits in other barley studies (Table 4). Poorter et al (submitted) studied a large number of physiological and morphological traits under near to optimal conditions, using the same $\mathrm{F}_{3}$ lines, marker data and linkage map as in the present study. This allows a comparison of positions and support intervals of QTLs. Comparisons with other studies can be made only at the chromosome level. In all, 15 QTLs appeared, in at least one environment, at the same chromosome as in other barley studies (Table 4). Eight of these had a support interval overlapping with QTLs of Poorter et al (submitted). The additive effects of the QTLs, detected at an identical chromosome in both studies, all had the same sign. One of these was a SLA-QTL on chromosome 3 that has also been detected in three other studies (Table 4). Yin et al (1999a, b) found this SLA-QTL in many different plant stages and in a single stage (flowering time) in different years. In the present study, it turned up at both nutrient levels. Thus, if indeed this SLA-QTL on chromosome 3 represents the same locus in all these studies, its expression seems to be hardly influenced by the environment. Whether it is really the same locus, however, requires more research. For example, fine-scale mapping and/or a candidate gene approach would need to be followed up (Faris et al, 1999).

Thus, as expected, some of the QTLs in the present study confirm earlier results in barley, but new QTLs were also found. A QTL detected in a certain cross may not be detected in another cross because the parents of the second cross carry identical alleles at that particular locus. Additionally, the expression of a QTL may depend on the genetic background, which may vary from one pair of parents to the next (Stam, 1998). However, more overlap was expected with the results of Poorter et al (submitted), as that study involved progeny from the same cross. The environment is known to influence QTL expression (Paterson et al, 1991; Lu et al, 1996; Zhuang et al, 1997; Yin et al, 1999b; Teulat et al, 2001). The conditions in the study of Poorter et al may have been substantially different from those in the present study, as they grew the plants in hydroponics. Thus, minor QTLs in one environment (or QTL study) may appear to be major ones in another environment (or QTL study). Beavis (1994) describes the same situation in maize: several studies done on the same traits in the same cross reported only partial overlap in the QTLs found. He ascribes this to the small number of progeny $(<500)$ tested, and states that only a small fraction of the QTLs was detected.

Table 4 Comparison of QTLs found in the present study to QTLs detected in other studies

\begin{tabular}{|c|c|c|c|c|c|c|c|}
\hline \multicolumn{4}{|c|}{ QTLs in the present study } & \multirow{2}{*}{$\begin{array}{c}\text { QTLs in Poorter et al } \\
\text { Chrom }\end{array}$} & \multicolumn{3}{|c|}{ QTLs in other studies (H. vulgare) } \\
\hline Trait & Nutr & Chrom & Pos & & Reference & Trait & Chrom \\
\hline LAR & High & 4 & 5.3 & 4 & & & \\
\hline \multirow[t]{3}{*}{ SLA } & Low & $3 a$ & $52.6^{*}$ & & Yin et al, 1999a & SLA, different stages & 3,4 \\
\hline & & 4 & 23.7 & & Yin et al, 1999b & SLA at flowering, different years & 3,4 \\
\hline & High & $3 a$ & $52.6^{*}$ & & & & \\
\hline LMF & High & 4 & $26.4^{*}$ & 4 & & & \\
\hline \#tillers & High & 4 & 42.8 & & Teulat et al, 2001 & \# fertile tillers, different testing sites & 4 \\
\hline \multirow[t]{3}{*}{ \#leaves } & Low & 1 & $3.0^{*}$ & & Teulat et al, 1997 & \# leaves on main tiller & $1,6,7$ \\
\hline & High & 6 & 15.7 & & & & \\
\hline & & 7 & 14.3 & & & & \\
\hline \multirow[t]{3}{*}{ Leafl } & Low & $2 a$ & 1.7 & $2 \mathrm{a}$ & & & \\
\hline & & 4 & 5.0 & 4 & & & \\
\hline & High & 1 & $31.4^{*}$ & 1 & & & \\
\hline Leafw & Low & $2 a$ & $1.7^{*}$ & $2 a$ & & & \\
\hline \multirow[t]{2}{*}{ Seedm } & - & 5 & 56.4 & & Teulat et al, 2001 & 1000 grain mass, different testing sites & 5,6 \\
\hline & & 6 & 8.1 & & & & \\
\hline
\end{tabular}

QTLs are mentioned only if these were detected at the same chromosome in the present study as in at least one other study. Traits, nutrient levels and chromosomes are coded as in Figure 2. Nutr = nutrient level in the present study (in other studies no different nutrient levels were applied), Chrom $=$ chromosome and Pos = position at the chromosome (in cM). ${ }^{*}=$ overlap between support intervals of QTL in the present study and the QTL in Poorter et al (submitted). 


\section{Association among traits}

Given the multiplicative nature of the growth analysis formulae, one would expect to find QTLs for different RGR components at the same position. Indeed, QTLs for LAR and LMF coincided, and the additive effects had the same sign. This type of association appears inevitably, since LAR is the product of LMF and SLA. Actually, one would expect more of these coinciding QTLs. An example is the QTL for RGR on chromosome 6 (11.7 cM, both nutrient levels). A QTL for NAR, LAR or both was expected at the same position. For NAR, only a small, nonsignificant peak (low nutrient: LOD 1.17, high nutrient: LOD 1.45) was found and for LAR no effect at all. For LMF, a peak was found at the high nutrient level $(5.0 \mathrm{cM}$, LOD 2.43), although, as this did not result in a peak for LAR, there was no effect on RGR. Perhaps, with a higher power, QTLs for NAR or LMF would have been detected.

Additionally, pleiotropic effects might exist among SLA, LMF and NAR, although these are not connected directly by the equations of the growth analysis. An indication for this was found only on chromosome 4, where a QTL for SLA at the low nutrient level shows overlap with a QTL for LMF and LAR at high nutrient conditions, all with a negative additive effect. If this were the same gene, it would imply that under low nutrient conditions the 'Mehola' allele had a positive effect on SLA and no effect on LMF (and thus LAR), whereas under high nutrient conditions the 'Mehola' allele did not influence SLA but increased LMF. No physiological explanation is apparent and it would require very accurate finemapping to assess whether pleiotropy is the case here.

Substantial overlap was found between QTLs for RGR and components on the one hand and morphological traits on the other hand. It is interesting to find overlapping QTLs for RGR and seed mass, since differences in RGR among natural populations could be largely explained by differences in initial seed mass (Elberse et al, 2003): plants growing from lighter seeds had a higher RGR than those growing from heavier seeds. The additive effect of the RGR-QTLs on chromosome 6 is negative, whereas the additive effect for the coinciding seed mass QTL is positive. This negative relationship supports previous results (Elberse et al, 2003) and indicates close linkage or possibly pleiotropic action of the underlying gene(s).

\section{Influence of nutrient level on QTLs}

In general, different QTLs were detected for the same trait under different nutrient conditions. It is quite common to find different QTLs for the same trait in different environments (Paterson et al, 1991; Lu et al, 1996; Zhuang et al, 1997; Yin et al, 1999b; Teulat et al, 2001). However, in the present study, it is clear that focusing only on the significant QTLs may be somewhat misleading, as in most cases the additive effect, at the position of the LOD peak, of a QTL in one environment and of the same map position in the other environment, had the same sign (Figure 3). The environmental influence on these QTLs might be gradual, for example, as a result of different amounts of gene product in different environments. Of course, lack of power might also explain why a QTL was found in one environment and a nonsignificant peak showing an additive effect with the same sign in the other. QTLs with a significant effect in one environment but virtually no effect in the other were also found. This could be attributable to the presence of on/off switches at these QTLs. No QTLs were found for which the effect of an allele in one environment is clearly opposite to that in the other. The positive correlation between additive effects in both environments fits the general lack of differences in plasticity found for RGR and components (Table 1A, see also Elberse et al, 2003).

QTLs for which the 'Mehola' alleles had a positive effect on the trait mean in both environments were RGR and LMF, as well as the number of tillers, leaves and roots. These results agree quite well with Elberse et al (2003), where different populations and accessions were compared. They found a high RGR and high number of leaves and tillers for the 'Mehola' population, but a low number of roots. Additionally, they found a high SLA for 'Mehola', while in the present study a positive 'Mehola' effect on SLA was only found under low nutrient conditions and a positive effect of the 'Ashqelon' allele was found in both environments (Figure 3). It is not surprising that the results of both studies do not completely agree, since in Elberse et al (2003) results for a broader spectrum of barley genotypes are presented. Moreover, mean trait values were used, so the joint effect of all loci that have an influence on these traits. A high number of loci are thought to underlie these traits, of which, most likely, only a subset has been detected in the present analysis. Of course, a plant having a high mean value for a certain character does not necessarily have the positive allele at all loci influencing this trait. The number of leaves presents a good example. 'Mehola' usually has a higher number of leaves than 'Ashqelon' (Elberse et al, 2003). Still, a QTL was revealed for which the 'Ashqelon' allele had a positive effect. Similarly, Alonso-Blanco et al (1998) detected QTLs for flowering time in Arabidopsis, where alleles of one of the parents at two QTLs caused earliness and at the other two QTLs generated lateness.

One might argue that differences, observed between the high and low nutrient conditions, might be explained by a difference in developmental stage of the plants. Yan et al (1998) and Yin et al (1999a) found different QTLs for one trait in different developmental stages of the plants. However, based on a prior study (Elberse et al, 2003), the harvest dates in the present study were chosen in a way that for both nutrient levels the plants were in the exponential growing phase. Thus, differences in developmental stage are not expected to play an important role in the present results.

\section{QTLs and genotype $\times$ environment interactions}

Concerning growth analysis components, QTLs having different phenotypic effects depending on nutrient supply were only detected for LAR, LMF and RGR. This partly corresponds to previous results (Elberse et al, 2003), where differences in plasticity among populations were found for LAR and LMF, but not for RGR. These results were interpreted as representing a trade-off in the degree of plasticity between NAR and LAR, resulting in a buffering of RGR from changes in plasticity. Populations that were most plastic for LAR were least plastic for 
NAR and vice versa. A similar trade-off was observed in the present QTL study. The Pearson correlation coefficient between the mean plasticity of NAR and LAR of the $\mathrm{F}_{3}$ lines was $-0.55(P<0.0001, n=140)$. At the QTL level, however, this trade-off was not detected. The plasticity QTL on chromosome 3a shows a positive additive effect for RGR plasticity and almost so for NAR plasticity (LOD 2.48, not shown), but no effect on LAR plasticity. The LAR-plasticity QTLs on chromosome 2a and chromosome 5 do not affect RGR and NAR plasticity, and thus offer no explanation for the trade-off either.

Although the results showed considerable variation, QTLs for plasticity were generally found in regions with a QTL at one of the nutrient levels only. This suggests that either their gene expression is regulated in response to environmental conditions, or that the phenotypic effect of gene expression depends on other aspects, such as plant size, or nutrient status. These possibilities cannot be distinguished, but it is clear that this pattern of variation has an impact on the evolution of phenotypic plasticity: such QTLs facilitate the fine-tuning of phenotypic responses, and reduce the impact of correlated selection responses across environments.

\section{Acknowledgements}

We thank Eviatar Nevo for supplying seed material and support, Ab Wijlhuizen for technical assistance, Hans Peter Koelewijn for advice on experimental set-up and statistics and Gerard Koorevaar and Koen Verhoeven for the valuable discussions on QTL analysis. Furthermore, Hendrik Poorter, Koen Verhoeven and Brian Forster are thanked for their comments on previous versions of this manuscript. The Earth and Life Science Foundation (ALW) of the Netherlands Organisation for Scientific Research (NWO) supported this study financially.

\section{References}

Alonso-Blanco C, El-Din El-Assal S, Coupland G, Koornneef M (1998). Analysis of natural allelic variation at flowering time loci in the Landsberg erecta and Cape Verde islands ecotypes of Arabidopsis thaliana. Genetics 149: 749-764.

Beavis WD (1994). The power and deceit of QTL experiments: lessons from comparative QTL studies. Proceedings of the 49th Annual Corn and Sorghum Industry Research Conference. American Seed Trade Association: Washington, DC, USA, pp 250-266.

Becker J, Vos P, Kuiper M, Salamini F, Heun M (1995). Combined mapping of AFLP and RFLP markers in barley. Mol Gen Genet 249: 65-73.

Brown AHD, Zohary D, Nevo E (1978). Outcrossing rates and heterozygosity in natural populations of Hordeum spontaneum Koch in Israel. Heredity 41: 49-62.

Chapin III FS (1991). Integrated responses of plants to stress. Bioscience 41: 29-36.

Elberse IAM, van Damme JMM, van Tienderen PH (2003). Plasticity of growth characteristics in wild barley (Hordeum spontaneum) in response to nutrient limitation. J Ecol 91: 371-382.

Evans GC (1972). The Quantitative Analysis of Plant Growth. Blackwell Scientific Publications: Oxford, UK.

Falconer DS, Mackay TFC (1996). Introduction to Quantitative Genetics. Longman Group Ltd: Harlow, UK.

Faris JD, Li WL, Liu DJ, Chen PD, Gill BS (1999). Candidate gene analysis of quantitative disease resistance in wheat. Theor Appl Genet 98: 219-225.
Grime JP, Hunt R (1975). Relative growth-rate: its range and adaptive significance in a local flora. J Ecol 63: 393-422.

Gutterman Y, Corbineau F, Côme D (1996). Dormancy of Hordeum spontaneum caryopses from a population on the Negev Desert Highlands. J Arid Environ 33: 337-345.

Hunt R, Cornelissen JHC (1997). Components of relative growth rate and their interrelations in 59 temperate plant species. New Phytol 135: 395-417.

Jansen RC, Stam P (1994). High resolution of quantitative traits into multiple loci via interval mapping. Genetics 136: 1447-1455.

Lambers H, Chapin III FS, Pons TL (1998). Plant Physiological Ecology. Springer: New York.

Lu C, Shen L, Tan Z, Xu Y, He P, Chen Y et al (1996). Comparative mapping of QTLs for agronomic traits of rice across environments using a doubled haploid population. Theor Appl Genet 93: 1211-1217.

Lynch M, Walsh B (1999). Genetic Analysis of Quantitative Traits. Sinauer: Sunderland.

Mauricio R (2001). Mapping quantitative trait loci in plants: uses and caveats for evolutionary biology. Nature 2: 370-381.

Nevo E (1992). Origin, evolution, population genetics and resources for breeding of wild barley, Hordeum spontaneum. In: Shewry PR (ed) The Fertile Crescent Barley: Genetics, Biochemistry, Molecular Biology and Biotechnology. CAB International: Wallingford, UK, pp 19-43.

Nevo E, Beiles A, Gutterman Y, Storch N, Kaplan D (1984). Genetic resources of wild cereals in Israel and vicinity II Phenotypic variation within and between populations of wild barley, Hordeum spontaneum. Euphytica 33: 737-756.

Nilan RA (1964). The Cytology and Genetics of Barley, 1951-1962. Washington State University: Pullman, USA.

Paterson AH, Damon S, Hewitt JD, Zamir D, Rabinowitch HD, Lincoln SE et al (1991). Mendelian factors underlying quantitative traits in tomato - comparison across species, generations, and environments. Genetics 127: 181-197.

Paterson AH, Lander ES, Hewitt JD, Peterson S, Lincoln SE, Tanksley SD (1988). Resolution of quantitative traits into Mendelian factors by using a complete linkage map of restriction fragment length polymorphisms. Nature 335: 721-726.

Poorter H, Nagel O (2000). The role of biomass allocation in the growth response of plants to different levels of light, $\mathrm{CO}_{2}$ : nutrients and water: a quantitative review. Austr J Plant Physiol 27: 595-607.

Poorter H, Remkes C (1990). Leaf area ratio and net assimilation rate of 24 wild species differing in relative growth rate. Oecologia 83: 553-559.

Poorter H, van Rijn CPE, Vanhala TK, Verhoeven KJF, de Jong YEM, Stam $P$ et al. A genetic analysis of relative growth rate and underlying components in Hordeum spontaneum. Oecologia. (submitted).

Powell W, Thomas WTB, Baird E, Lawrence P, Booth A, Harrower B et al (1997). Analysis of quantitative traits in barley by the use of amplified fragment length polymorphisms. Heredity 79: 48-59.

Qi X, Stam P, Lindhout P (1998). Use of locus-specific AFLP markers to construct a high-density molecular map in barley. Theor Appl Genet 96: 376-384.

Ramsay L, Macaulay M, Ivanissevich S, MacLean K, Cardle L, Fuller J et al (2000). A simple sequence repeat-based linkage map of barley. Genetics 156: 1997-2005.

Reynolds HL, D'Antonio C (1996). The ecological significance of plasticity in root weight ratio in response to nitrogen. Plant Soil 185: 75-97.

Shipley B, Peters H (1990). A test of the Tilman model of plant strategies: relative growth rate and biomass partitioning. Am Natur 136: 139-153.

Stam P (1998). Crop physiology, QTL analysis and plant breeding. In: Lambers $\mathrm{H}$, Poorter $\mathrm{H}$, Van Vuuren MMI (eds) Inherent Variation in Plant Growth: Physiological 
Mechanisms and Ecological Consequences. Backhuys Publishers: Leiden, The Netherlands, pp 429-440.

Teulat B, Merah O, Souyris I, This D (2001). QTLs for agronomic traits from a Mediterranean barley progeny grown in several environments. Theor Appl Genet 103: 774-787.

Teulat B, Monneveux P, Wery J, Borries C, Souyris I, Charrier A et al (1997). Relationships between relative water content and growth parameters under water stress in barley: a QTL study. New Phytol 137: 99-107.

Van Ooijen JW (1999). LOD significance thresholds for QTL analysis in experimental populations of diploid species. Heredity 83: 613-624.

Van Rijn CPE, Heersche I, Van Berkel YEM, Nevo E, Lambers H, Poorter H (2000). Growth characteristics in Hordeum spontaneum populations from different habitats. New Phytol 146: 471-481.

Via S, Gomulkiewicz R, De Jong G, Scheiner SM, Schlichting CD, Van Tienderen PH (1995). Adaptive phenotypic plasticity: consensus and controversy. Trends Ecol Evol 10: 212-217.
Yan JQ, Zhu J, He CX, Benmoussa M, Wu P (1998). Quantitative trait locus analysis for the developmental behavior of tiller number in rice (Oryza sativa L). Theor Appl Genet 97: 267-274.

Yin X, Kropff MJ, Stam P (1999a). The role of ecophysiological models in QTL analysis: the example of specific leaf area in barley. Heredity 82: 415-421.

Yin X, Stam P, Dourleijn CJ, Kropff MJ (1999b). AFLP mapping of quantitative trait loci for yield-determining physiological characters in spring barley. Theor Appl Genet 99: 244-253.

Zhuang JY, Lin HX, Lu J, Qian HR, Hittalmani S, Huang N et al (1997). Analysis of QTL $\times$ environment interaction for yield components and plant height in rice. Theor Appl Genet 95 799-808.

Zohary D (1969). The progenitors of wheat and barley in relation to domestication and agricultural dispersal in the Old World. In: Ucko PJ, Dimbleby GW (eds) The Domestication and Exploitation of Plants and Animals. Duckworth: London, UK, pp 47-66. 